\title{
APPLICATION OF ANISOTROPIC THIN PLATE THEORY FOR DEVELOPMENT OF DISPLACEMENTS FOR SILICON <111> CRYSTALS PLATES IN CYLINDRICAL SYSTEMS
}

\author{
A. JAVIDINEJAD \\ Department of Mechanical and Aerospace Engineering \\ University of Texas at Arlington \\ Arlington, TX 96019 USA \\ E-mail: amir.javidinejad@gmail.com
}

\begin{abstract}
Silicon $<111>$ crystal is one of the crystal orientations, which shows potential for application in micro device developments of pressure diaphragms for measurement purposes. To date, no theoretical basis has been examined to develop the deflections for computational analysis purposes [1]. This paper presents the development of the diaphragm deflections for Silicon $<111>$ Crystal in Cylindrical coordinates system. The Silicon $<111>$ crystal possesses transverse isotropic properties. Thus, an anisotropic thin plate theory is used here to develop the plate deflection. A numerical example is given to compare the theoretical results with Finite Element Analysis (FEA) results.
\end{abstract}

Key words: transverse isotropic, thin plate elasticity, FEA, pressure diaphragm, anisotropic.

\section{Introduction}

Many of the micro pressure measurement devices utilize silicon wafers as the substrate pressure diaphragm $[2,3,4,5]$. These single crystalline silicon structures have different mechanical properties with respect to crystalline orientation due to the fact that single-crystalline silicon is an anisotropic material. In order to take advantages of the plane isotropic properties of the silicon crystalline, the $<111>$ wafer can be used. Single-crystal silicon micro devices fabricated using $<111>$ silicon would be least sensitive to device orientation with respect to crystallographic orientations. This paper intends to develop the closed-form solution of the circular diaphragm deflection using the classical theory of plate elasticity.

\section{Silicon mechanical properties}

Silicon wafers can be selected as the base material model for this design for pressure transducer devices. At room temperature, Silicon $(\mathrm{Si})$ is a metallic gray crystalline material [6] with a low density of $2330\left(\mathrm{Kg} / \mathrm{m}^{3}\right)$. It has a cubic crystal structure which is identical to diamond. Silicon wafers cut parallel to the $<111>$ plane have "transverse isotropic" properties. This $<111>$ wafer plane cut would be used as the structural modeling base to simplify the material aspects of the Silicon. Figure 1 illustrates the silicon cubic structure with the crystallographic coordinate system shown 


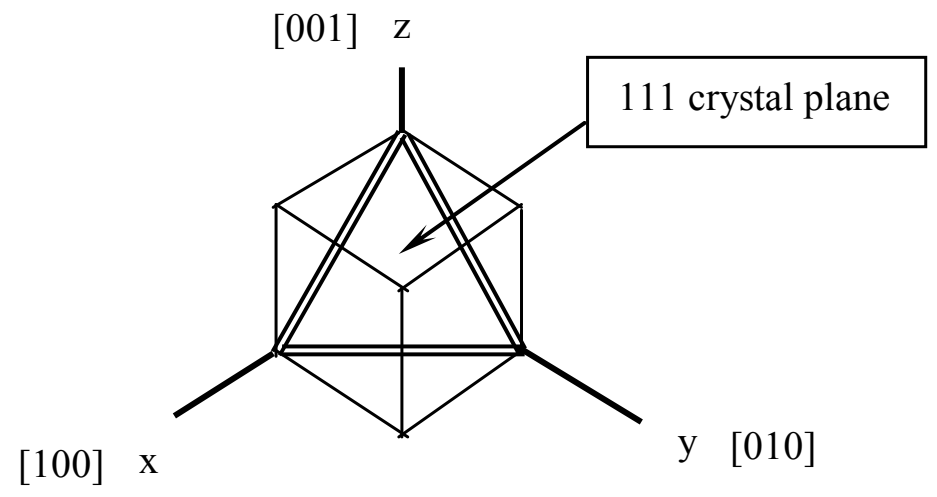

Fig.1. Silicon crystal structure.

The mechanical properties of the $<111>$ plane silicon are shown in Tab.1.

Table 1. Silicon $<111>$ Cut Mechanical Properties.

\begin{tabular}{ll}
\hline$E_{11}=E_{22}$ & $1.697 \times 10^{11}(\mathrm{~Pa})$ \\
$E_{33}$ & $1.883 \times 10^{11}(\mathrm{~Pa})$ \\
$v_{12}=v_{21}$ & 0.265 \\
$v_{23}$ & 0.447 \\
$v_{32}$ & 0.237 \\
$v_{13}$ & 0.184 \\
$v_{31}$ & 0.165 \\
$G_{12}$ & $0.671 \times 10^{11}(\mathrm{~Pa})$ \\
$G_{23}=G_{31}$ & $0.580 \times 10^{11}(\mathrm{~Pa})$
\end{tabular}

\section{Plate theory}

In the small deflection plate theory in cylindrical coordinate systems, the displacement components in the radial $(r)$ and circumferential $(\theta)$ directions can be expressed as follows [7]

$$
u=-z \frac{\partial w}{\partial r}, \quad v=-\frac{z}{r} \frac{\partial w}{\partial \theta}, \quad w=w(r, \theta)
$$

whereas, $u$ is the radial displacement, $v$ is the tangential displacement, and $w$ is the vertical displacement.

Also, the strain-displacement components are defined as 


$$
\begin{aligned}
& \varepsilon_{r}=\frac{\partial u}{\partial r}, \\
& \varepsilon_{\theta}=\frac{u}{r}+\frac{1}{r} \frac{\partial v}{\partial \theta}, \\
& \varepsilon_{z}=\frac{\partial w}{\partial z}, \\
& \gamma_{\theta z}=\frac{\partial v}{\partial z}+\frac{1}{r} \frac{\partial w}{\partial \theta}, \\
& \gamma_{r z}=\frac{\partial w}{\partial r}+\frac{\partial u}{\partial z}, \\
& \gamma_{r \theta}=\frac{1}{2}\left(\frac{1}{r} \frac{\partial u}{\partial \theta}+\frac{\partial v}{\partial r}-\frac{v}{r}\right) .
\end{aligned}
$$

These strain-displacement components can be rewritten in terms of the vertical deflection $w$.

By substitution of Eq.(3.1) into the first, second and the sixth equations in Eq.(3.2) set, the strain components can be expressed in terms of the mid plane deflection $w$

$$
\begin{aligned}
& \varepsilon_{r}=-z \frac{\partial^{2} w}{\partial r^{2}}, \\
& \varepsilon_{\theta}=-\frac{z}{r} \frac{\partial w}{\partial r}-\frac{z}{r^{2}} \frac{\partial^{2} w}{\partial \theta^{2}}, \\
& \gamma_{r \theta}=\frac{z}{r^{2}} \frac{\partial w}{\partial \theta}-\frac{z}{r} \frac{\partial^{2} w}{\partial r \partial \theta} .
\end{aligned}
$$

The stress-strain relations from Hooke's law are as follows

$$
\begin{aligned}
& \varepsilon_{r}=\frac{1}{E_{r}} \sigma_{r}-\frac{v_{\theta r}}{E_{\theta}} \sigma_{\theta}, \\
& \varepsilon_{\theta}=-\frac{v_{r \theta}}{E_{r}} \sigma_{r}+\frac{1}{E_{\theta}} \sigma_{\theta}, \\
& \gamma_{r \theta}=\frac{1}{G_{r \theta}} \tau_{r \theta} .
\end{aligned}
$$

The differential equations of the equilibrium are 


$$
\begin{aligned}
& \frac{\partial \sigma_{r}}{\partial r}+\frac{1}{r} \frac{\partial \tau_{r \theta}}{\partial \theta}+\frac{\partial \tau_{r z}}{\partial z}+\frac{\sigma_{r}-\sigma_{\theta}}{r}=0, \\
& \frac{\partial \tau_{r \theta}}{\partial r}+\frac{1}{r} \frac{\partial \sigma_{\theta}}{\partial \theta}+\frac{\partial \tau_{\theta z}}{\partial z}+\frac{2 \tau_{r \theta}}{r}=0 \\
& \frac{\partial \tau_{r z}}{\partial r}+\frac{1}{r} \frac{\partial \tau_{\theta z}}{\partial \theta}+\frac{\partial \sigma_{\theta z}}{\partial z}+\frac{\tau_{r z}}{r}=0 .
\end{aligned}
$$

Further, by substituting Eqs (3.3) into Eq.(3.4) and using the equilibrium Eqs (3.5), the stress components can be solved. These stress components in the final form are as follows

$$
\begin{aligned}
& \sigma_{r}=-z \frac{E_{r}}{1-v_{r \theta} v_{\theta r}}\left[\frac{\partial^{2} w}{\partial r^{2}}+v_{\theta r}\left(\frac{1}{r} \frac{\partial w}{\partial r}+\frac{1}{r^{2}} \frac{\partial^{2} w}{\partial \theta^{2}}\right)\right], \\
& \sigma_{\theta}=-z \frac{E_{\theta}}{1-v_{r \theta} v_{\theta r}}\left[v_{r \theta} \frac{\partial^{2} w}{\partial r^{2}}+\frac{1}{r} \frac{\partial w}{\partial r}+\frac{1}{r^{2}} \frac{\partial^{2} w}{\partial \theta^{2}}\right], \\
& \tau_{r \theta}=-2 z G_{r \theta} \frac{\partial^{2}}{\partial r \partial \theta}\left(\frac{w}{r}\right), \\
& \tau_{\theta z}=-\frac{1}{2}\left(\frac{h^{2}}{4}-z^{2}\right)\left[\frac{E_{r} v_{\theta r}+2 G_{r \theta}\left(1-v_{r} v_{\theta}\right)}{1-v_{r \theta} v_{\theta r}}\left(\frac{1}{r} \frac{\partial^{3} w}{\partial r^{2} \partial \theta}\right)+\frac{E_{\theta}}{1-v_{r \theta} v_{\theta r}}\left(\frac{1}{r^{2}} \frac{\partial}{\partial \theta}\left(\frac{\partial w}{\partial r}+\frac{1}{r} \frac{\partial^{2} w}{\partial \theta^{2}}\right)\right)\right], \\
& \tau_{r z}=-\frac{1}{2}\left(\frac{h^{2}}{4}-z^{2}\right)\left[\begin{array}{l}
\left.\frac{E_{r} v_{\theta r}+2 G_{r \theta}\left(1-v_{r} v_{\theta}\right)}{1-v_{r \theta} v_{\theta r}}\left(\frac{1}{r^{2}} \frac{\partial^{2}}{\partial \theta^{2}}\left(\frac{\partial w}{\partial r}-\frac{w}{r}\right)\right)+\frac{E_{r}}{1-v_{r \theta} v_{\theta r}}\left(\frac{\partial^{3} w}{\partial r^{3}}+\frac{1}{r} \frac{\partial^{2} w}{\partial r^{2}}\right)\right] \\
-\frac{E_{\theta}}{1-v_{r \theta} v_{\theta r}} \frac{1}{r^{2}}\left(\frac{\partial w}{\partial r}+\frac{1}{r^{2}} \frac{\partial^{2} w}{\partial \theta^{2}}\right)
\end{array}\right]
\end{aligned}
$$

(For the thin plate theory, $\sigma_{z}$ is much smaller than the other stress components and is negligible.)

The moment and shear stress resultants can be evaluated by integrating the stress components over the thickness, as follows

$$
\begin{aligned}
& M_{r}=\int_{-h / 2}^{h / 2} \sigma_{r} z d z, \\
& M_{\theta}=\int_{h / 2}^{h / 2} \sigma_{\theta} z d z, \\
& M_{r \theta}=\int_{-h / 2}^{h / 2} \tau_{r \theta} z d z,
\end{aligned}
$$




$$
\begin{aligned}
& Q_{r}=\int_{-h / 2}^{h / 2} \tau_{r z} d z \\
& Q_{\theta}=\int_{h / 2}^{h / 2} \tau_{\theta z} d z, \\
& M_{r}=\frac{-E_{r} h^{3}}{12\left(1-v_{r \theta} v_{\theta r}\right)}\left[\frac{\partial^{2} w}{\partial r^{2}}+v_{\theta r}\left(\frac{1}{r} \frac{\partial w}{\partial r}+\frac{1}{r^{2}} \frac{\partial^{2} w}{\partial \theta^{2}}\right)\right] \\
& M_{\theta}=\frac{-E_{\theta} h^{3}}{12\left(1-v_{r \theta} v_{\theta r}\right)}\left[v_{r \theta} \frac{\partial^{2} w}{\partial r^{2}}+\frac{1}{r} \frac{\partial w}{\partial r}+\frac{1}{r^{2}} \frac{\partial^{2} w}{\partial \theta^{2}}\right], \\
& M_{r \theta}=-\frac{G_{r \theta} h^{3}}{6} \frac{\partial^{2}}{\partial r \partial \theta}\left(\frac{w}{r}\right), \\
& N_{\theta}=-\left[\frac{\left(E_{r} v_{\theta r}+2 G_{r \theta}\left(1-v_{r} v_{\theta}\right)\right) h^{3}}{12\left(1-v_{r \theta} v_{\theta r}\right)}\left(\frac{1}{r} \frac{\partial^{3} w}{\partial r^{2} \partial \theta}\right)+\frac{E_{\theta} h^{3}}{12\left(1-v_{r \theta} v_{\theta r}\right)}\left(\frac{1}{r^{2}} \frac{\partial}{\partial \theta}\left(\frac{\partial w}{\partial r}+\frac{1}{r} \frac{\partial^{2} w}{\partial \theta^{2}}\right)\right)\right], \\
& N_{r}=-\left[\begin{array}{l}
\frac{\left(E_{r} v_{\theta r}+2 G_{r \theta}\left(1-v_{r} v_{\theta}\right)\right) h^{3}}{12\left(1-v_{r \theta} v_{\theta r}\right)}\left(\frac{1}{r^{2}} \frac{\partial^{2}}{\partial \theta^{2}}\left(\frac{\partial w}{\partial r}-\frac{w}{r}\right)\right)+\frac{E_{r} h^{3}}{12\left(1-v_{r \theta} v_{\theta r}\right)}\left(\frac{\partial^{3} w}{\partial r^{3}}+\frac{1}{r} \frac{\partial^{2} w}{\partial r^{2}}\right) \\
-\frac{E_{\theta} h^{3}}{12\left(1-v_{r \theta} v_{\theta r}\right)} \frac{1}{r^{2}}\left(\frac{\partial w}{\partial r}+\frac{1}{r^{2}} \frac{\partial^{2} w}{\partial \theta^{2}}\right)
\end{array}\right] .
\end{aligned}
$$

For a plate element, the governing force equilibrium differential equation in the $z$-direction in cylindrical coordinates would be

$$
\frac{\partial N_{r}}{\partial r}+\frac{1}{r} N_{r}+\frac{1}{r} \frac{\partial N_{\theta}}{\partial \theta}+q=0
$$

Similarly, as previously by substitution of transverse shear loading shown by Eqs (3.9) ( $N_{r}$ and $N_{\theta}$ ) into the governing force equilibrium differential Eq.(3.10) in the $z$-direction in cylindrical coordinates, the differential equation for the deflection of an anisotropic plate would be shown as

$$
\begin{aligned}
& D_{r} \frac{\partial^{4} w}{\partial r^{4}}+2 D_{r \theta} \frac{1}{r^{2}} \frac{\partial^{4} w}{\partial r^{2} \partial \theta^{2}}+D_{\theta} \frac{1}{r^{4}} \frac{\partial^{4} w}{\partial \theta^{4}}+2 D_{r} \frac{1}{r} \frac{\partial^{3} w}{\partial r^{3}}+2 D_{r \theta} \frac{1}{r^{3}} \frac{\partial^{3} w}{\partial r \partial \theta^{2}}+ \\
& +D_{\theta} \frac{1}{r^{2}} \frac{\partial^{2} w}{\partial r^{2}}+2\left(D_{\theta}+D_{r \theta}\right) \frac{1}{r^{4}} \frac{\partial^{2} w}{\partial \theta^{2}}+D_{\theta} \frac{1}{r^{3}} \frac{\partial w}{\partial r}=q
\end{aligned}
$$

where the rigidity coefficients are defined as follows 


$$
\begin{aligned}
& D_{r}=\frac{E_{r} h^{3}}{12\left(1-v_{r \theta} v_{\theta r}\right)}, \\
& D_{\theta}=\frac{E_{\theta} h^{3}}{12\left(1-v_{r \theta} v_{\theta r}\right)}, \\
& D_{k}=\frac{G_{r \theta} h^{3}}{12}, \\
& D_{r \theta}=D_{r} v_{\theta r}+2 D_{k} .
\end{aligned}
$$

In the case of silicon $<111>$ wafer, where you have plane isotropy and,

$$
\begin{aligned}
& E=E_{r}=E_{\theta}, \\
& v=v_{r \theta}=v_{\theta r}, \\
& G_{r \theta}=\frac{E_{r}}{2\left(1+v_{r \theta}\right)}=\frac{E_{\theta}}{2\left(1+v_{\theta r}\right)},
\end{aligned}
$$

the bending and twisting rigidities shown by Eqs (3.12) would all be equal. The coefficient $D_{r \theta}$ likewise would become

$$
D_{r \theta}=\frac{E_{r} h^{3}}{12\left(1-v_{r \theta} v_{\theta r}\right)} v_{\theta r}+2 \frac{G_{r \theta} h^{3}}{12},
$$

after the substation of the bending rigidity $D_{r}$. Further, by substitution of $\frac{E_{r}}{2\left(1+v_{r \theta}\right)}$ for shear modulus, $G_{r \theta}$ in Eq.(3.14) one would have

$$
D_{r \theta}=\frac{E_{r} h^{3}}{12\left(1-v_{r \theta} v_{\theta r}\right)} v_{\theta r}+\frac{E_{r} h^{3}}{12\left(1+v_{r \theta}\right)}=\frac{E_{r} h^{3}}{12\left(1-v_{r \theta} v_{\theta r}\right)}
$$

Hence, one would have

$$
D=D_{r}=D_{\theta}=D_{r \theta}=\frac{E_{r} h^{3}}{12\left(1-v^{2}\right)}
$$

Substitution of the Eq.(3.16) above into the differential equation for deflection shown by Eq.(3.11) would simplify the derivation of a solution for deflection for the $<111>$ plane silicon wafer. 


$$
\begin{aligned}
& \frac{\partial^{4} w}{\partial r^{4}}+2 \frac{1}{r^{2}} \frac{\partial^{4} w}{\partial r^{2} \partial \theta^{2}}+\frac{1}{r^{4}} \frac{\partial^{4} w}{\partial \theta^{4}}+2 \frac{1}{r} \frac{\partial^{3} w}{\partial r^{3}}+2 \frac{1}{r^{3}} \frac{\partial^{3} w}{\partial r \partial \theta^{2}}+ \\
& +\frac{1}{r^{2}} \frac{\partial^{2} w}{\partial r^{2}}+4 \frac{1}{r^{4}} \frac{\partial^{2} w}{\partial \theta^{2}}+\frac{1}{r^{3}} \frac{\partial w}{\partial r}=\frac{q}{D}
\end{aligned}
$$

Equation (3.17) is the Laplacian equation

$$
\nabla^{2}\left(\frac{\partial^{2} w}{\partial r^{2}}+\frac{1}{r} \frac{\partial w}{\partial r}+\frac{1}{r^{2}} \frac{\partial^{2} w}{\partial \theta^{2}}\right)=\frac{q}{D} \quad \text { or } \quad \nabla^{4} w=\frac{q}{D} .
$$

Under the assumption that the distributed loading is applied symmetrically over the diaphragm surface and the boundary conditions are also symmetric, then the surface deflection of the diaphragm would also be symmetric. Thus, one can assume that the deflection $w$, is independent of $\theta$. Hence, Eq.(3.18) would reduce to

$$
\nabla^{2}\left(\frac{\partial^{2} w}{\partial r^{2}}+\frac{1}{r} \frac{\partial w}{\partial r}\right)=\frac{q}{D}
$$

likewise,

$$
\begin{aligned}
& N_{r}=-D \frac{d}{d r}\left(\nabla^{2} w\right)=-D \frac{d}{d r}\left(\frac{\partial^{2} w}{\partial r^{2}}+\frac{1}{r} \frac{\partial w}{\partial r}\right) \\
& N_{\theta}=0 .
\end{aligned}
$$

After performing integration of Eq.(3.19) the equation for the plate deflection would become

$$
w(r)=\frac{q r^{4}}{64 D}+A r^{2} \ln r+B r^{2}+C \ln r+F .
$$

For a circular diaphragm that is fixed at the circumferential boundary edge, the following boundary conditions are applicable
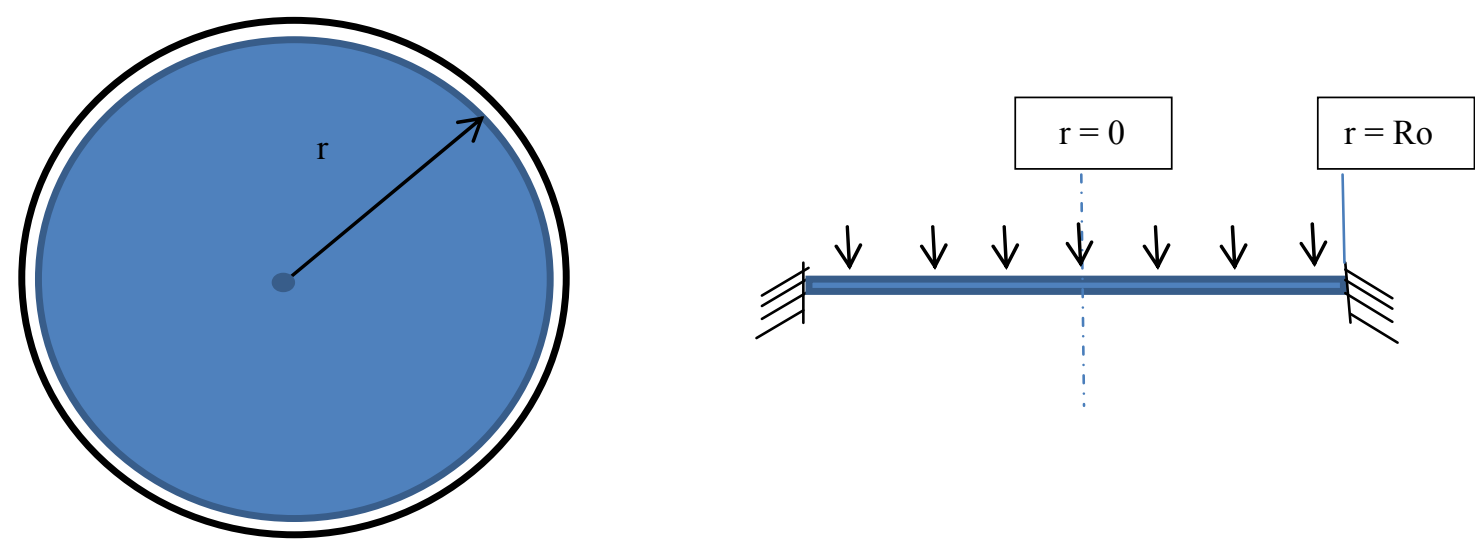

Fig.2. The schematics of a circular diaphragm. 


$$
\begin{aligned}
& w=0 \quad \text { at } \quad r=R o, \\
& \frac{d w}{d r}=0 \quad \text { at } \quad r=R o, \\
& -2 \pi R o N_{r}=q \pi R o^{2} \quad \text { at } \quad r=R o, \\
& \frac{d w}{d r}=0 \quad \text { at } \quad r=0 .
\end{aligned}
$$

By substitution of the boundary condition (3.25) into Eq.(3.20) one would have

$$
N_{R o}=\frac{-q R o}{2}=\frac{-q R o}{2}-\frac{4 A D}{R o} .
$$

This yields that coefficient $A$ is zero $(A=0)$. Using boundary condition (3.26) and substituting it into the derivative of Eq.(3.22) one would have the following

$$
\left.\frac{d w}{d r}\right|_{r=0}=\frac{C}{r}+A r+2 B r+2 A r \ln (r)+\left.\frac{q r^{3}}{16 D}\right|_{r=0}=\frac{C}{r}+2 B r+\left.\frac{q r^{3}}{16 D}\right|_{r=0}=0 \Rightarrow C=0 .
$$
would have

Likewise, using boundary condition (3.24) and substituting it into the derivative of Eq.(3.22) one

$$
\left.\frac{d w}{d r}\right|_{r=R o}=\frac{C}{r}+A r+2 B r+2 A r \ln (r)+\left.\frac{q r^{3}}{16 D}\right|_{r=R o}=2 B r+\left.\frac{q r^{3}}{16 D}\right|_{r=R o}=0 \Rightarrow B=\frac{-q R o^{2}}{32 D}
$$

Also, using boundary condition (3.23) and substituting in into Eq.(3.22) one would have

$$
\begin{aligned}
& \left.\frac{d w}{d r}\right|_{r=R o}=\frac{q r^{4}}{64 D}+A r^{2} \ln (r)+B r^{2}+C \ln (r)+\left.F\right|_{r=R o}=\frac{q r^{4}}{64 D}+\frac{-q R o^{2}}{32 D} r^{2}+\left.F\right|_{r=R o}=0 \Rightarrow \\
& \Rightarrow F=\frac{q R o^{4}}{64 D} .
\end{aligned}
$$

Thus to develop the closed-form solution of the deflection, Eq.(3.22) representing the clamped circular diaphragm deflection by application of the coefficients becomes

$$
w(r)=\frac{q r^{4}}{64 D}+\frac{-q R o^{2}}{32 D} r^{2}+\frac{q R o^{4}}{64 D} .
$$

\section{Numerical analysis}

Assume a circular diaphragm 40 microns thick $(h)$ with a radius of 800 microns $(R o)$. Also assume a uniform distributed pressure of $689.5 \mathrm{~Pa}(q)$ applied on the diaphragm. The deflection response versus the 
radius of this diaphragm, due to the application of $689.5 \mathrm{~Pa}$ of pressure is plotted in Fig.3. The data provided is from the closed-form solution of Eq.(3.31) and also the FEA analysis of the same $<111>$ silicon diaphragm. Since the diaphragm is clamped at the boundary edges one would observe that the deflection at the boundary goes to zero and the deflection at the center of the diaphragm is maximum. Figures 4 and 5 illustrate the FEA simulation of the same $<111>$ silicon diaphragm with appropriate material properties.

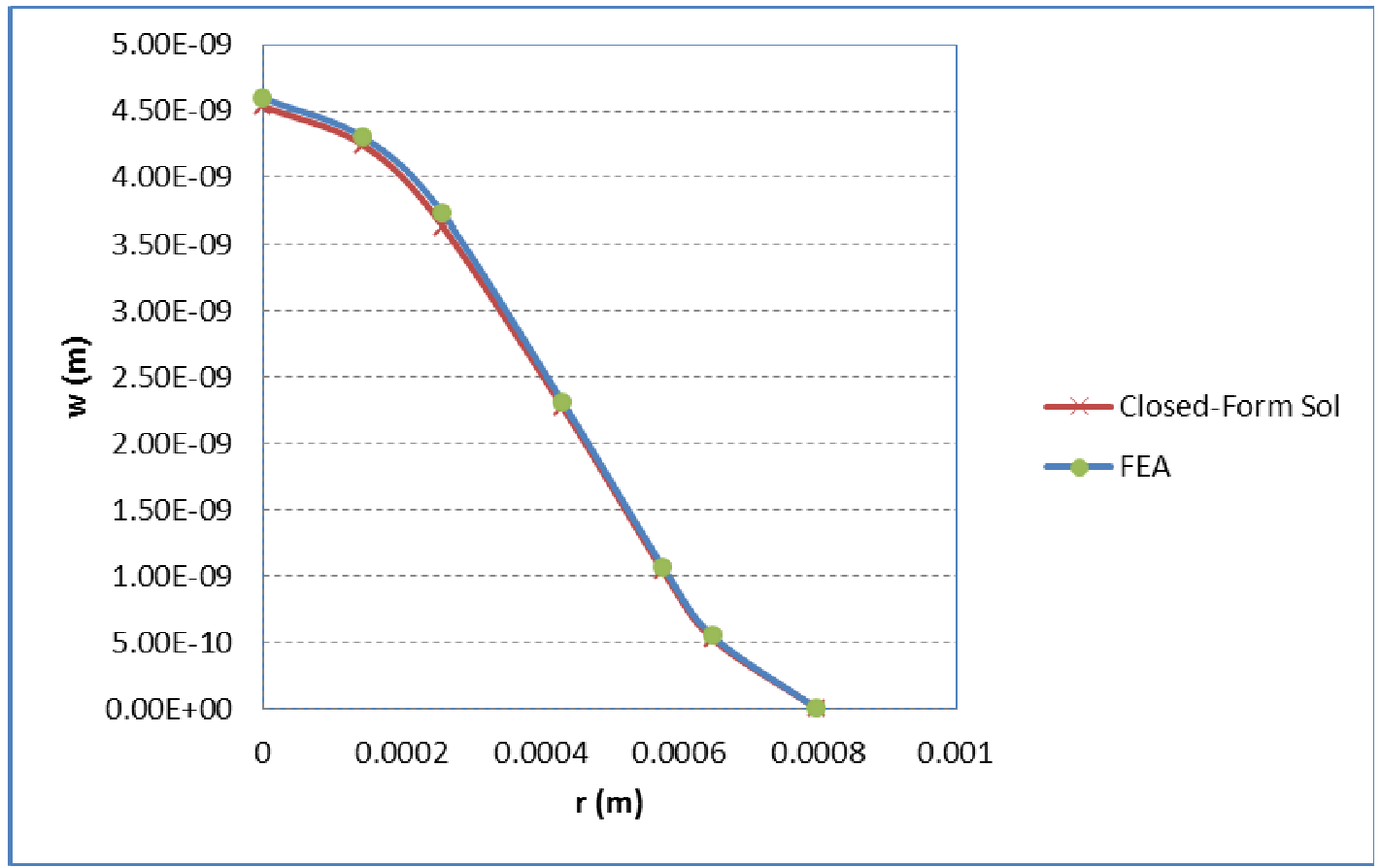

Fig.3. The $<111>$ Silicon Diaphragm Deflection vs. Radius.

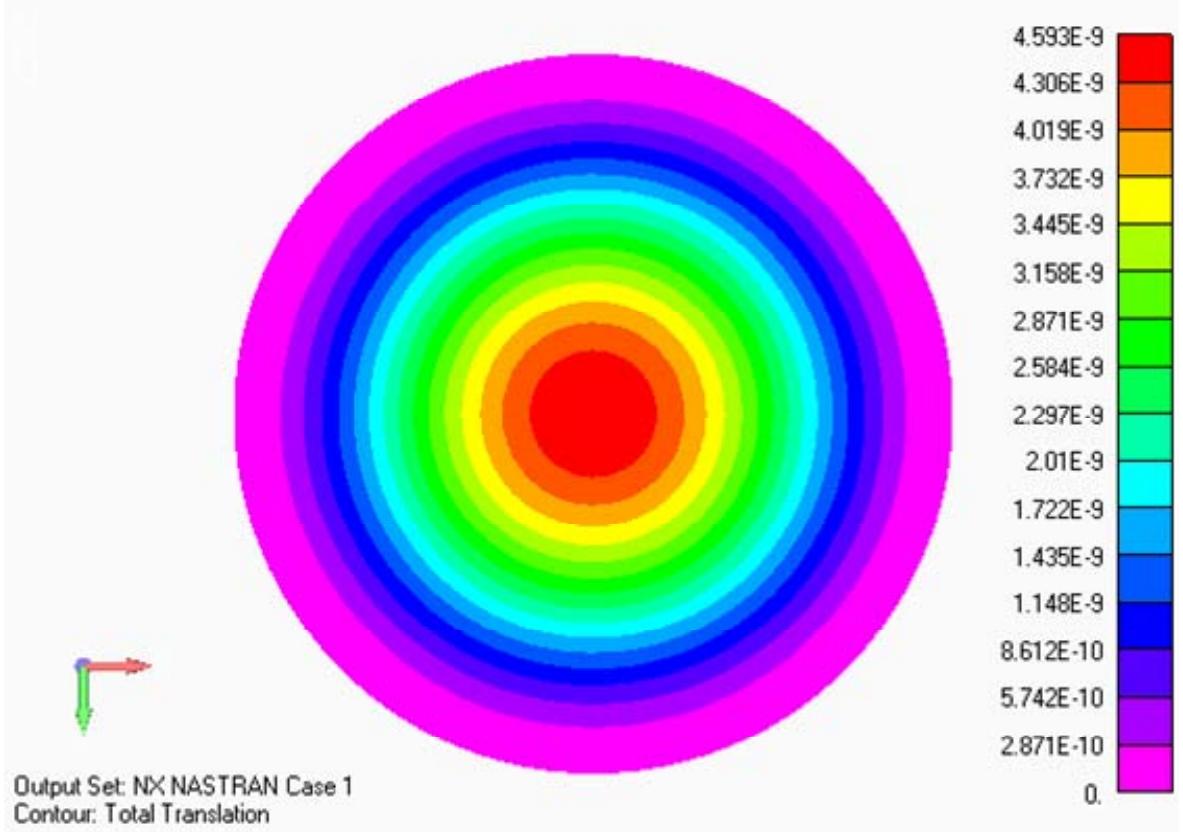

Fig.4. FEA Simulation of the $<111>$ Silicon Diaphragm (Top View). 


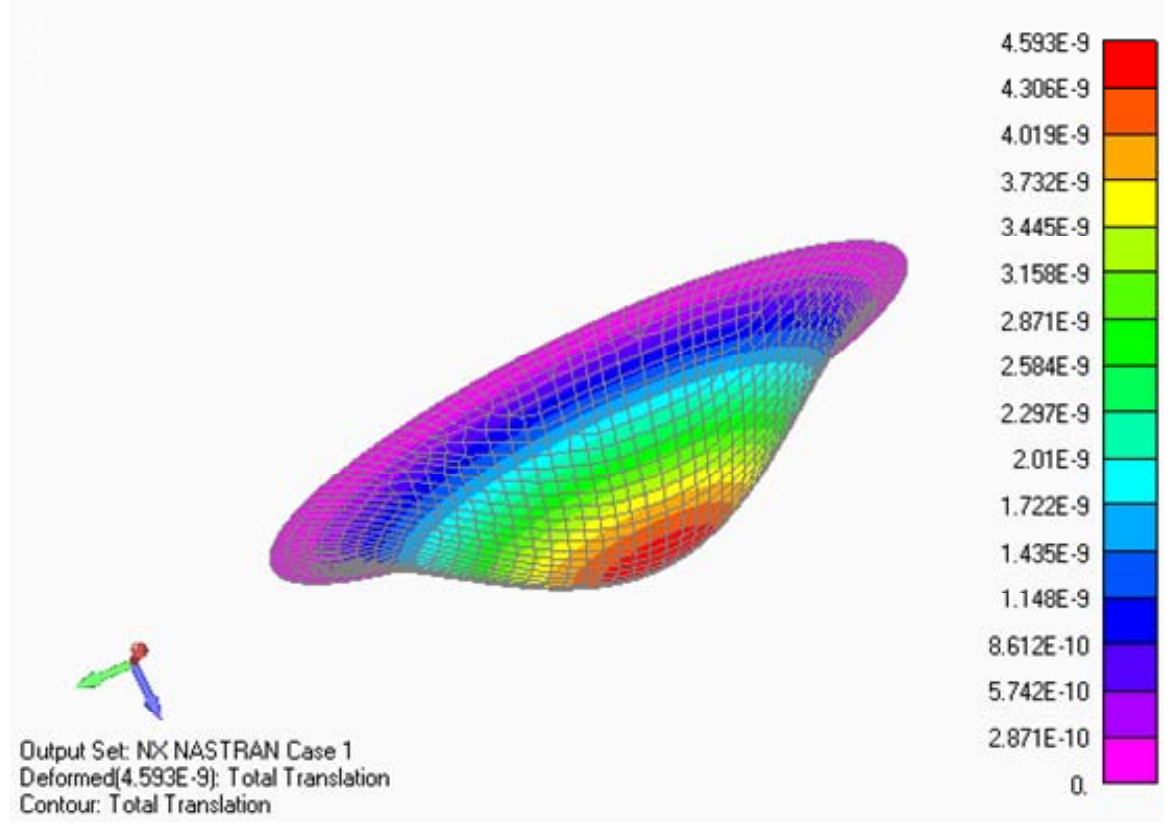

Fig.5. FEA Simulation of the $<111>$ Silicon Diaphragm (Side View).

The stress analysis for the diaphragm by using an axisymmetric Finite Element Analysis shows the following results for radial and tangential stresses in the $<111>$ silicon circular diaphragm.

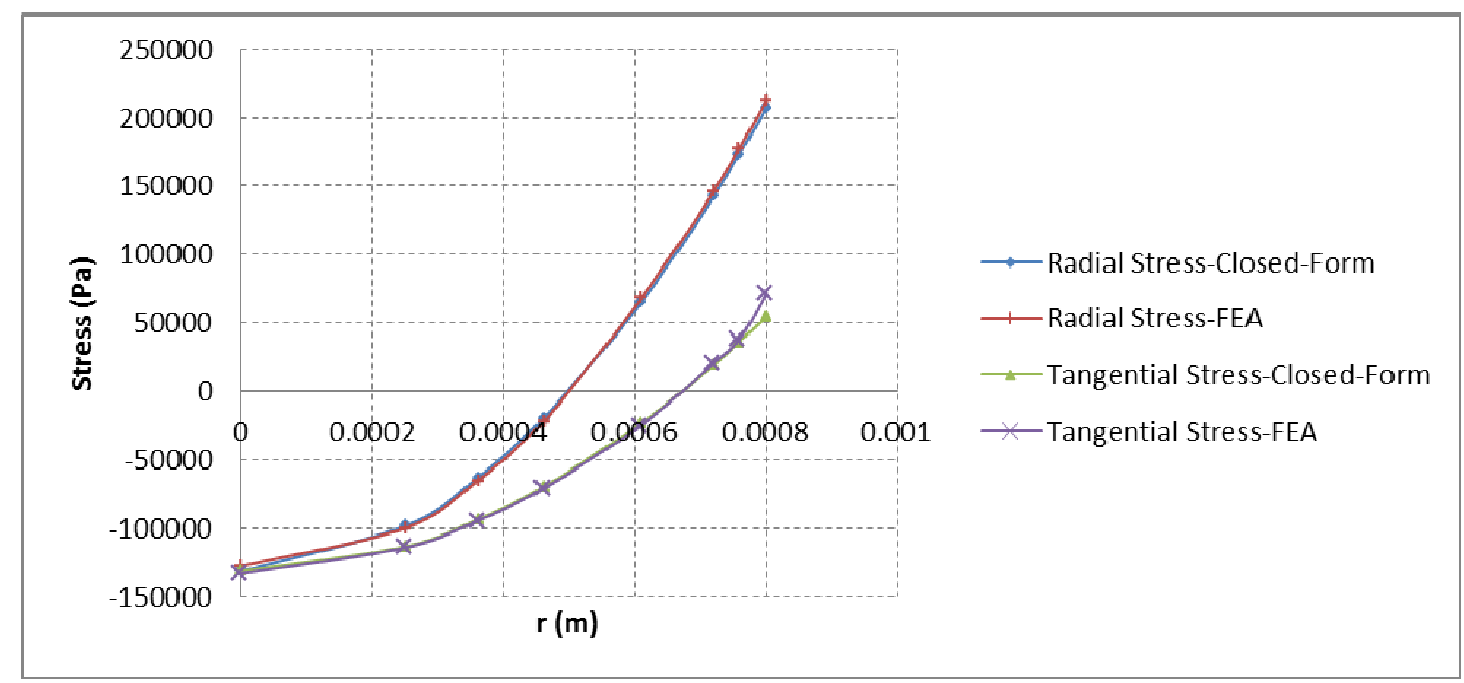

Fig.6. The $<111>$ Silicon Diaphragm Radial and Tangential Stress vs. Radius. 

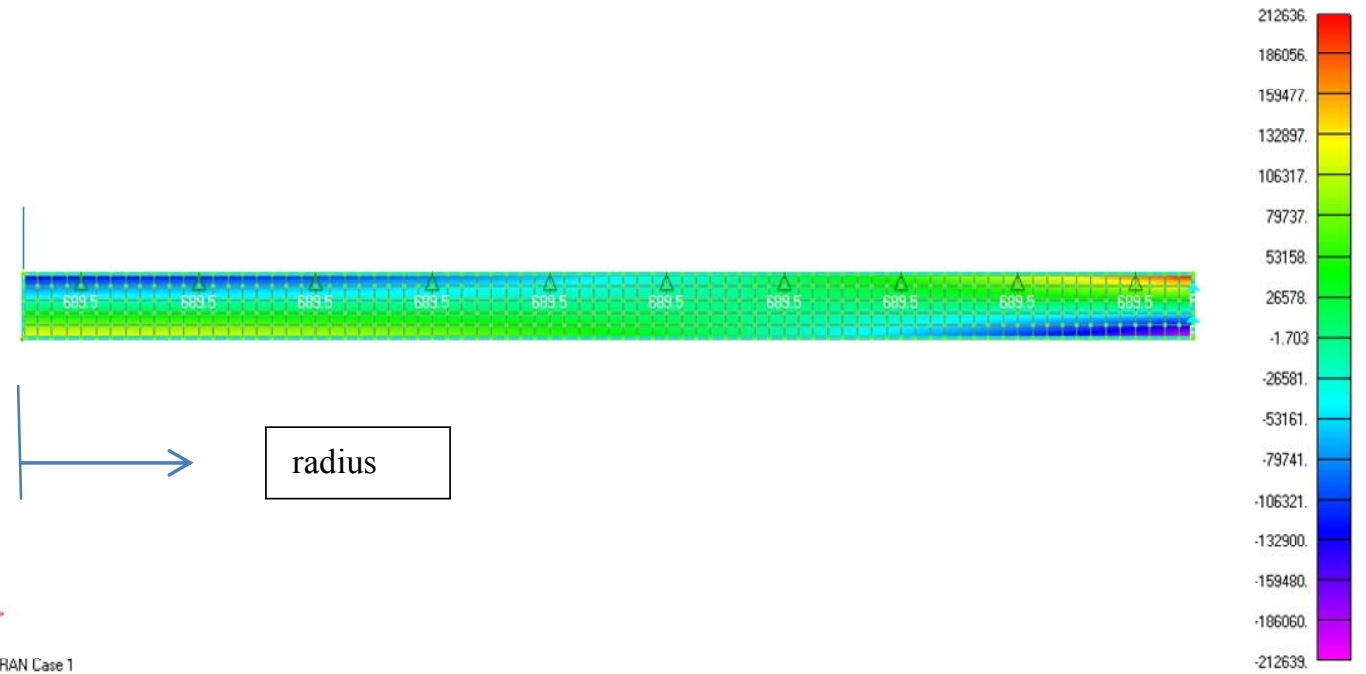
Output Set NX NASTRAN Case 1
Contour: Axisym Radial Stress

Fig.7. The Axisymmetric $<111>$ Silicon Diaphragm Model Radial Stress vs. Radius.
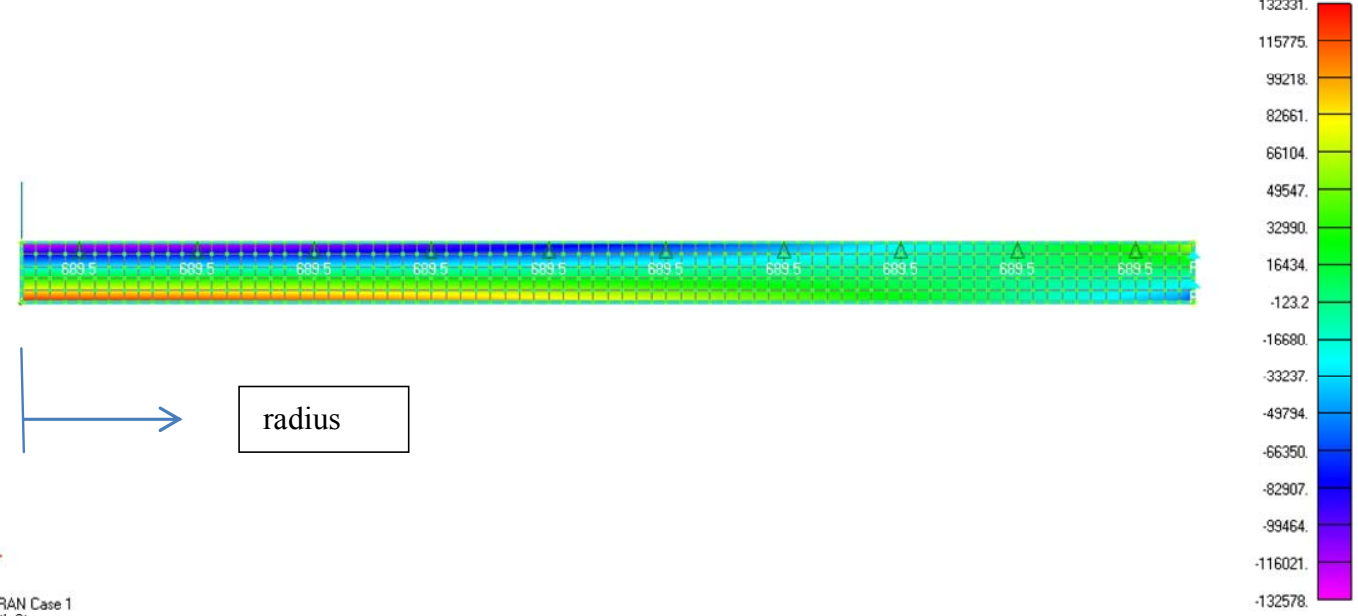

Output Set NX NASTRAN Case

Fig.8. The Axisymmetric $<111>$ Silicon Diaphragm Model Tangential Stress vs. Radius.

\section{Conclusions}

Classical theories of plate elasticity were used to develop the pressure-deflection closed form solution for the $<111>$ silicon crystal diaphragm. Plane isotropic properties of the $<111>$ silicon were utilized. A clamped circular diaphragm was modeled in order to determine the coefficients of the deflection equation of the diaphragm via anisotropic deflection equation. FEA analysis, with anisotropic material properties of the $<111>$ silicon inputted in the FEA package, was performed. A comparison was drawn between the closed form solution and the FEA results for the deflection of the diaphragm to establish the accuracy of the closed form solution. Also, a comparison was mode between the closed form solution and the FEA results for the radial and tangential stresses of the diaphragm to establish the accuracy of the closed form solution. The results of the closed-form theoretical and numerical analysis match perfectly with great accuracy. Thus, this paper has presented the accurate development of the diaphragm deflections for silicon $<111>$ crystal in polar coordinates system. 


\section{Nomenclature}

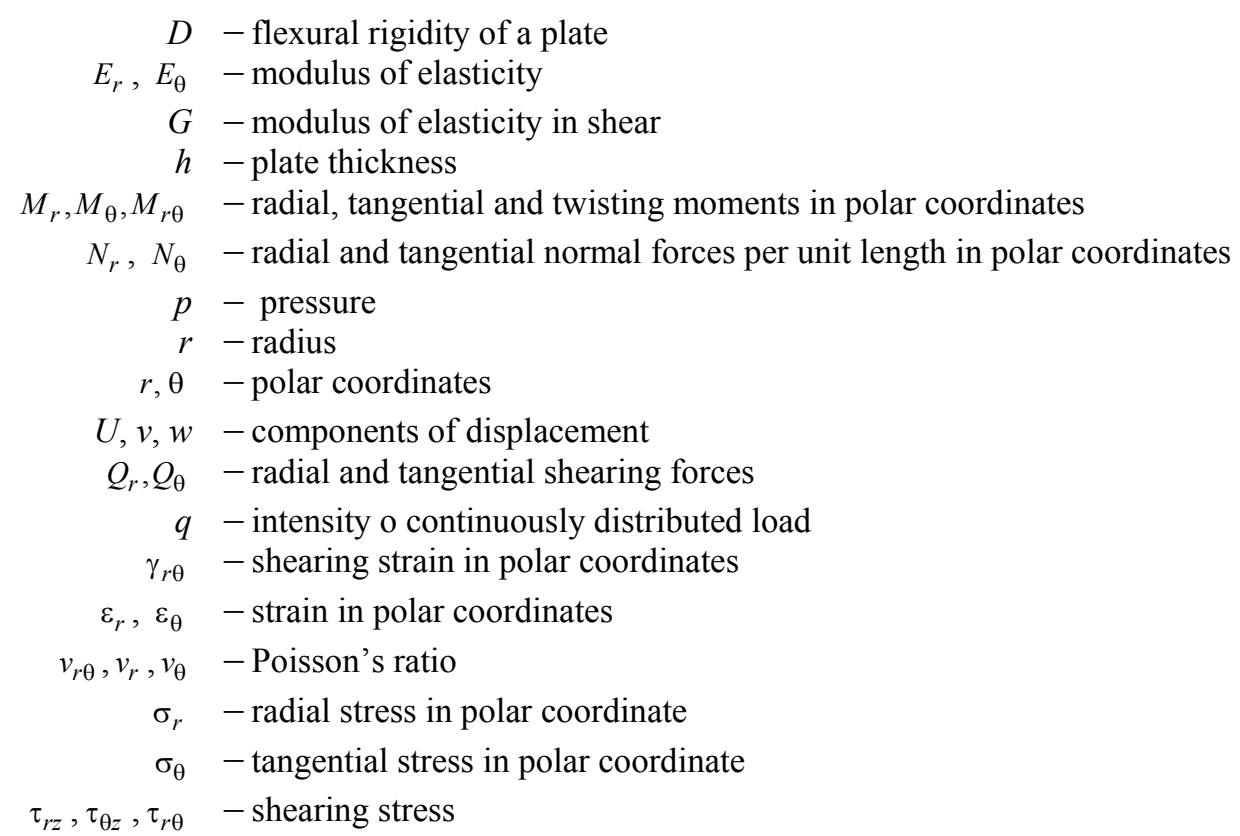

\section{References}

[1] Javidinejad A. (1999): Mechanical Design of Embeddable Micro-Smart Sensing Systems. - Ph.D. Dissertation, UTA.

[2] Yang J. (2006): An Introduction to the Theory of Piezoelectricity. - London, U.K: Springer Science Publications.

[3] Fumio S. (2012): Silicon Semiconductor Crystal Technology. - Elsevier Science Publishing.

[4] Lee S., Park S., Kim J., Yi S. and Cho D. (2002): Surface/Bulk micromachined single-crystalline silicon microgyroscope. - IEEE/ASME Journal of Microelectromechanical Systems.

[5] Bell T.E., Gennissen P.T.J., DeMunter D. and Kuhl M. (1996): Porous silicon as a sacrificial material. - IOP Journal of Micromechcal and Microengineering.

[6] Buschow K.H. Jürgen, Cahn Robert W., Flemings Merton C., Ilschner B., Kramer Edward J., Mahajan S. and Veyssière P. (2011): Encyclopedia of Materials Science and Engineering. - Elsevier Ltd Publishing.

[7] Aghalovyan L. (2015): Asymptotic Theory of Anisotropic Plates and Shells. - World Scientific Publishing Co Pte Ltd, Armenia. 